\title{
Evaluation of Peripheral Arterial Disease with Nonenhanced Quiescent-Interval Single-Shot MR Angiography ${ }^{1}$
}

Philip A. Hodnett, MD

loannis Koktzoglou, PhD

Amir H. Davarpanah, MD

Timothy G. Scanlon, MD

Jeremy D. Collins, MD

Eugene E. Dunkle, RT

Navyash Gupta, MD

James C. Carr, MD

Robert R. Edelman, MD
John J. Sheehan, MD
Materials and Methods:

Results:

Conclusion:
To assess the diagnostic performance of quiescent-interval single-shot (QISS) magnetic resonance (MR) angiography, a nonenhanced two-dimensional electrocardiographically gated single-shot balanced steady-state free precession examination for the evaluation of symptomatic chronic lower limb ischemia.

For this prospective institutional review board-approved, HIPAA-compliant study, the institutional review board waived the requirement for informed patient consent. The QISS nonenhanced MR angiography technique was evaluated in a two-center trial involving 53 patients referred for lower extremity MR angiography for suspected or known chronic peripheral arterial disease (PAD), with contrast material-enhanced MR angiography serving as the noninvasive reference standard. The accuracy of stenosis assessments performed with the nonenhanced MR angiography sequence was evaluated relative to the reference standard. Per-segment, per-region, and per-limb sensitivities and specificities were calculated, and assessments were considered correct only if they were in exact agreement with the reference standard-derived assessments. Generalized estimating equation (GEE) modeling with use of an unstructured binomial logit analysis was used to account for clustering of multiple measurements per case. The sensitivity and specificity of QISS MR angiography for the determination of nonsignificant $(<50 \%)$ versus significant $(50 \%-100 \%)$ stenosis were compared with the sensitivity and specificity of the reference standard.

${ }^{1}$ From the Departments of Radiology (P.A.H., I.K., J.J.S., E.E.D., R.R.E.) and Vascular Surgery (N.G.), NorthShore University HealthSystem, 2650 Ridge Ave, Evanston, IL 60201; and Department of Radiology, Feinberg School of Medicine, Northwestern University, Chicago, III (P.A.H. A.H.D., T.G.S., J.D.C., J.C.C.). Received July 5, 2010; revision requested August 24; revision received December 8; accepted December 29; final version accepted March 2, 2011 Supported in part by a grant from the Grainger Foundation. Address correspondence to R.R.E. (e-mail: redelman @northshore.org).

${ }^{\circ}$ RSNA, 2011
The diagnostic performance of nonenhanced MR angiography was found to be nearly equivalent to the diagnostic performances of contrast-enhanced MR angiography and digital subtraction angiography. Non-GEE segment-based analysis revealed that for the two reviewers, nonenhanced MR angiography had sensitivities of $89.7 \%$ (436 of 486 segments) and $87.0 \%$ (423 of 486 segments) and specificities of $96.5 \%$ (994 of 1030 segments) and $94.6 \%$ (973 of 1028 segments).

QISS nonenhanced MR angiography offers an alternative to currently used imaging tests for symptomatic chronic lower limb ischemia, for which the administration of iodinated or gadolinium-based contrast agents is contraindicated.

RSNA, 2011 
D eripheral arterial disease (PAD) is a potentially debilitating manifestation of systemic atherosclerosis and is associated with increased risks of myocardial infarction, stroke, and cardiovascular disease-related death $(1,2)$. The accurate diagnosis of PAD is important for managing the disease and because it contributes to prognostic information regarding increased mortality risk.

Ankle brachial index measurement is a sensitive test for hemodynamically significant PAD (3) and can be supplemented by Doppler ultrasonography (US). Additional imaging may be performed if revascularization is being considered, when the ankle brachial index results are indeterminate, or if the patient is unable to exercise after a rest study yields negative results. Imaging options include duplex US, computed tomographic (CT) angiography, contrast material-enhanced magnetic resonance (MR) angiography, and digital subtraction angiography (DSA).

The reported sensitivity of CT angiography for the detection of greater than $50 \%$ stenosis is on the order of $89 \%-$ $100 \%$, with specificity ranging from $92 \%$ to $100 \%(3,4)$. However, peripheral CT angiography exposes patients to particularly high doses of ionizing radiation, and given the prevalence of renal dysfunction in patients with PAD, there is also concern about the risk of contrast agent-induced nephropathy (5).

Contrast-enhanced MR angiography enables one to accurately evaluate PAD while largely avoiding the risks of ionizing radiation and contrast agent-induced nephropathy $(6,7)$. A concern, however,

\section{Advances in Knowledge}

- Nonenhanced MR angiography performed by using the quiescentinterval single-shot (QISS) sequence enables accurate evaluation of the extent and severity of symptomatic chronic lower limb ischemia, with a typical imaging time of 6.4 minutes.

- QISS MR angiography is insensitive to patient motion and provides consistent image quality in the pelvis. is the risk of nephrogenic systemic fibrosis associated with the use of gadolinium based contrast agents in patients with impaired renal function. Concerns about nephrogenic systemic fibrosis in populations at increased risk for renal disease have dampened enthusiasm regarding contrast-enhanced MR angiography (8).

With nonenhanced MR angiography techniques, exposure to ionizing radiation is avoided and the potential risks from contrast agent-induced nephropathy and nephrogenic systemic fibrosis are eliminated. Although early time-offlight techniques for nonenhanced peripheral MR angiography are reasonably accurate (9), they have fallen into disuse because of long imaging times and poor image quality (10). The combination of recent technical advances and concerns about the risks of nephrogenic systemic fibrosis has prompted a resurgence of interest in nonenhanced MR angiography alternatives. Although newer techniques have emerged, they have been incompletely evaluated, with limited clinical assessment in symptomatic populations (11). Nonenhanced MR angiography performed by using an electrocardiographically gated fast spinecho sequence has been shown to enable accurate imaging of the calf and pedal arteries, but it requires optimal selection of systolic trigger delays to avoid image artifacts and erroneous stenosis estimations (12). Quiescentinterval single-shot (QISS) nonenhanced MR angiography does not require modification of the sequence based on the absence, presence, or severity of PAD. The purpose of our study was to assess the utility of QISS, a nonenhanced twodimensional electrocardiographically gated single-shot balanced steady-state

\section{Implication for Patient Care}

- QISS MR angiography represents an accurate alternative to contrastenhanced MR angiography for patients in whom abnormal renal function or hypersensitivity is a contraindication to the administration of gadolinium-based contrast agents. free precession acquisition sequence for the evaluation of symptomatic chronic lower limb ischemia.

\section{Materials and Methods}

In this prospective institutional review board-approved, Health Insurance Portability and Accountability Act-compliant study, a waiver of the requirement for patient consent was approved by the institutional review board. The QISS nonenhanced MR angiography technique (Fig 1) was evaluated in a two-center trial involving 53 patients who were referred for lower extremity MR angiography for suspected or known chronic PAD, with contrast-enhanced MR angiography as the noninvasive standard of reference. The accuracy of stenosis assessments performed by using the nonenhanced MR angiography sequence was evaluated relative to the reference standard, contrast-enhanced MR angiography. Assessments were considered to be correct only when they were in exact agreement with the assessments made by using the reference standard.

\section{Published online before print 10.1148/radiol.11101336 \\ Radiology 2011; 260:282-293 \\ Abbreviations: \\ $\mathrm{Cl}=$ confidence interval \\ DSA = digital subtraction angiography \\ $\mathrm{GEE}=$ generalized estimating equation \\ $\mathrm{GFR}=$ glomerular filtration rate \\ $\mathrm{PAD}=$ peripheral arterial disease \\ QISS = quiescent-interval single shot}

\section{Author contributions:}

Guarantor of integrity of entire study, P.A.H.; study concepts/study design or data acquisition or data analysis/ interpretation, all authors; manuscript drafting or manuscript revision for important intellectual content, all authors manuscript final version approval, all authors; literature research, P.A.H., I.K., A.H.D., J.J.S., J.C.C., R.R.E.; clinical studies, P.A.H., I.K., J.D.C., J.J.S., E.E.D., N.G., J.C.C.; statistical analysis, P.A.H., I.K., A.H.D., J.D.C.; and manuscript editing, P.A.H., I.K., A.H.D., J.D.C., E.E.D., N.G., J.C.C., R.R.E.

\section{Funding:}

This research was supported by the National Institutes of Health (grant R01HL096916).

Potential conflicts of interest are listed at the end of this article. 
Patient inclusion criteria were referral with symptoms attributable to chronic lower limb ischemia and evaluation with contrast-enhanced MR angiography. Study exclusion criteria were renal impairment, as indicated by a glomerular filtration rate (GFR) lower than $30 \mathrm{~mL} / \mathrm{min} / 1.73 \mathrm{~m}^{2}$, that precluded contrast-enhanced MR angiography, contraindications to MR imaging or other reasons for the inability to complete both the nonenhanced and the contrastenhanced MR angiography portions of the study, and previous arterial revascularization including stent placement. Between March 2009 and March 2010, 53 patients ( 37 men, 16 women; mean age, 66.1 years; age range, $24-87$ years) who were referred for evaluation of chronic lower limb ischemia underwent nonenhanced and contrast-enhanced MR angiography examinations. The men had a mean age of 64.6 years (age range, 24-87 years), and the women had a mean age of 69.8 years (age range, 4785 years). At presentation, 17 of these patients were found to have GFRs lower than $60 \mathrm{~mL} / \mathrm{min} / 1.73 \mathrm{~m}^{2}$.

A total of six patients met the exclusion criteria: four patients who had chronic renal impairment, as indicated by a GFR lower than $30 \mathrm{~mL} / \mathrm{min} / 1.73 \mathrm{~m}^{2}$, that precluded contrast-enhanced MR angiography and two patients who were unable to complete nonenhanced and contrast-enhanced MR angiography because of pain at the time of imaging. No patients had an implanted stent or contraindications to MR imaging. Selective DSA was performed in 15 patients (290 arterial segments) during a therapeutic interventional procedure or when MR angiograms revealed pathologic conditions that warranted further investigation before these procedures.

\section{Imaging Protocols}

Imaging was performed by using a 1.5-T MR unit (Magnetom Avanto; Siemens Healthcare, Erlangen, Germany). The nonenhanced MR angiography technique performed was QISS (13). In brief, this is a two-dimensional electrocardiographically gated single-shot balanced steadystate free precession acquisition that involves the use of an initial in-plane

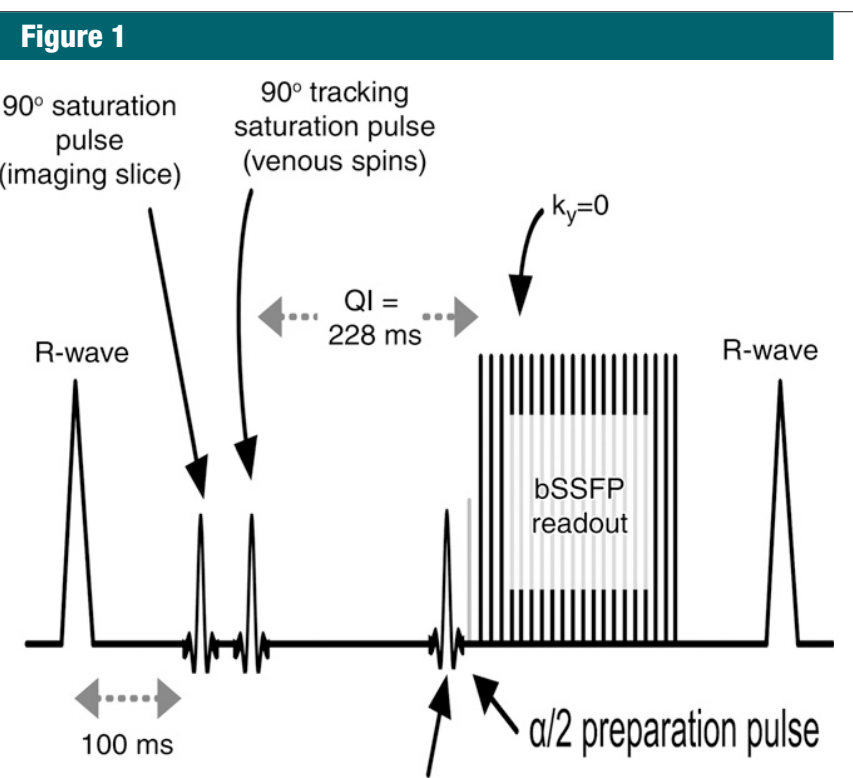

Fat saturation pulse

Figure 1: Diagram of QISS pulse sequence. A two-dimensional single-shot balanced steady-state free precession (bSSFP) pulse sequence is used to image arterial spins within the section during diastole, when flow is slow or absent. $k_{y}=$ phase-encoding line, $Q l=$ quiescent interval, $\alpha / 2=$ one half the radiofrequency excitation flip angle.

saturation pulse to suppress background tissues and a quiescent inflow period. The quiescent inflow period overlaps with the period of rapid systolic flow, ensuring maximal inflow of unsaturated spins into the section, even in the setting of very slow flow. Imaging parameters are fixed, regardless of the heart rate and other factors, without variation from patient to patient. The imaging parameters for nonenhanced MR angiography, stepping-table bolus-chase MR angiography, and time-resolved MR angiography of the calf are cited in Table 1 .

For the QISS nonenhanced MR angiography sequence, eight or nine groups of 48 sections were acquired to encompass the peripheral arteries, from the level of the infrarenal aorta to the pedal arteries. Assuming a typical heart rate of 72 beats per minute (R-R interval, $833 \mathrm{msec}$ ), the typical imaging time for nine stations at 48 sections per station was 6.4 minutes. The Avanto Magnetom MR imaging system was equipped with 32 radiofrequency receiver channels and a gradient system capable of a maximum gradient strength of $45 \mathrm{mT} / \mathrm{m}$ and a $200-\mu$ sec rise time of $0-45 \mathrm{mT} / \mathrm{m}$.
Multiple-element peripheral vascular coils and spine-array coils were used to receive the MR signal. Means of 6.11 unique coil elements \pm 2.85 (standard deviation) and 7.0 unique coil elements \pm 4.5 were positioned anterior and posterior to the body, respectively, across the nine QISS stations. The coil element sizes varied between 196 and $576 \mathrm{~cm}^{2}$. A 16-channel peripheral vascular coil, a six-channel body-array coil, and a fourchannel flex coil were placed anterior to the subject. In addition, a 24-channel spine-array coil was placed posterior to the subject.

The contrast-enhanced MR angiography protocol involved a dual-injection technique consisting of time-resolved contrast-enhanced MR angiography through the calf followed by steppingtable MR angiography encompassing the remainder of the peripheral arterial system (14). A time-resolved coronal acquisition (time-resolved imaging with stochastic trajectories, or TWIST) was performed through the calf and proximal region of the foot by using multiphase time-resolved angiography with an interleaved stochastic trajectories 
Table 1

\section{Parameters for Electrocardiographically Triggered Nonenhanced QISS and Contrast-enhanced MR Angiography Sequences}

\begin{tabular}{|c|c|c|c|}
\hline Parameter & Nonenhanced QISS & Contrast-enhanced Bolus Chase & $\begin{array}{l}\text { Contrast- } \\
\text { enhanced } \\
\text { Time resolved }\end{array}$ \\
\hline Acquisition time & 432 Cardiac cycles & $\begin{array}{r}15.5 \mathrm{sec} \text { in pelvic station, } 17.9 \\
\text { and19.6 sec in thigh station }\end{array}$ & $111 \mathrm{sec}$ \\
\hline Acquisition mode & $2 \mathrm{D}$ & $3 \mathrm{D}$ & $3 \mathrm{D}$ \\
\hline TR (msec) & 3.0 & 2.9 & 3.1 \\
\hline TE (msec) & 1.4 & 1.0 & 1.1 \\
\hline $\begin{array}{l}\text { Excitation angle } \\
\text { (degrees) }\end{array}$ & 90 & 25 & 25 \\
\hline Field of view $\left(\mathrm{mm}^{3}\right)^{\star}$ & $400 \times 260 \times 3$ & $\begin{array}{l}328 \times 500 \times 146 \text { in pelvic station, } \\
328 \times 500 \times 168 \text { in thigh station, } \\
344 \times 500 \times 125 \text { in calf station }\end{array}$ & $366 \times 450 \times 104$ \\
\hline Matrix $^{\dagger}$ & $400(800) \times 260(520)$ & $\begin{array}{l}227 \times 384 \times 64(104) \text { in pelvic } \\
\text { station, } 227 \times 384 \times 74(120) \\
\text { in thigh station, } 282 \times 512 \times \\
64(104) \text { in calf station }\end{array}$ & $\begin{array}{l}312 \times 512 \times \\
80(86)\end{array}$ \\
\hline $\begin{array}{l}\text { Spatial } \\
\text { resolution }\left(\mathrm{mm}^{3}\right)^{\dagger}\end{array}$ & $\begin{array}{l}1.0(0.5) \times 1.0(0.5) \\
\quad \times 3.0\end{array}$ & $\begin{array}{l}1.4 \times 1.3 \times 2.3(1.4) \text { in pelvic } \\
\text { and thigh stations, } 1.2 \times \\
1.0 \times 1.9(1.2) \text { in calf station }\end{array}$ & $\begin{array}{c}1.2 \times 0.9 \times \\
1.4(1.3)\end{array}$ \\
\hline $\begin{array}{l}\text { Section partial Fourier } \\
\text { factor }\end{array}$ & $\ldots$ & $6 / 8$ & $6 / 8$ \\
\hline $\begin{array}{l}\text { Phase partial Fourier } \\
\text { factor }\end{array}$ & $5 / 8$ & $6 / 8$ & $6 / 8$ \\
\hline $\begin{array}{l}\text { GRAPPA imaging } \\
\text { factor }{ }^{\ddagger}\end{array}$ & Two & Two & Three \\
\hline Bandwidth (Hz/pixel) & 658 & 444 & 444 \\
\hline Fat suppression & Yes & No & No \\
\hline
\end{tabular}

Note.-Nine stations, at 48 sections per station, were imaged by using the QISS nonenhanced sequence. With the time-resolved sequence, imaging was performed in 14 temporal phases, with an acquired temporal resolution of 23.2 seconds, and with a reconstructed temporal resolution of 5.8 seconds. GRAPPA = generalized autocalibrating partially parallel acquisition, $\mathrm{TE}=$ echo time, $\mathrm{TR}=$ repetition time, $3 \mathrm{D}=$ three-dimensional, $2 \mathrm{D}=$ two-dimensional

* Fields of view in the right-left $\times$ anterior-posterior $\times$ feet-head direction are cited.

† Values reconstructed after interpolation are in parentheses.

acquisition (15) and an injection of 5-8 $\mathrm{mL}$ of gadopentetate dimeglumine (Magnevist; Bayer, Berlin, Germany) or gadobenate dimeglumine (MultiHance; Bracco Imaging, Milan, Italy) at a rate of $2 \mathrm{~mL} / \mathrm{sec}$. For the stepping-table acquisition, a 1-2-mL test bolus was used to determine the timing. Three stations were imaged before and after the contrast material was administered with use of a dual-phase infusion protocol that involved the infusion of $0.14 \mathrm{mmol}$ of the contrast material per kilogram of body weight, initially at $1.5 \mathrm{~mL} / \mathrm{sec}$ and then at $0.6 \mathrm{~mL} / \mathrm{sec}$. The gadopentetate dimeglumine or gadobenate dimeglumine was administered intravenously and fol- lowed by a saline chaser. The total contrast agent dose typically was $0.2 \mathrm{mmol} / \mathrm{kg}$.

In 15 patients (290 arterial segments), one leg was examined with selective DSA. With use of a standard angiographic unit, selective DSA images were obtained from the thigh to the feet via a 5-F catheter positioned in the vessel of interest. Anteroposterior and lateral views were chosen, at the discretion of the interventionalist, to best depict the arteries and were obtained by using a field of view of $28-38 \mathrm{~cm}$.

\section{Image Analysis}

Two radiologists (P.A.H. and T.G.S.), each with at least 7 years experience reading MR angiograms, independently evaluated the nonenhanced and contrastenhanced MR angiograms while blinded to the patients' medical histories and clinical findings. The order of the images was randomized for the readings. The American College of Radiology grading system used in a previously reported multiinstitutional trial of peripheral MR angiography (7) was used to evaluate the degree of vessel stenosis: Individual readers reviewed the subtracted image data sets on maximal intensity projection images, multiplanar reformatted reconstructions, and axial source images that were available for subjective assessment of stenosis degree at an off-line workstation (Leonardo; Siemens Healthcare). A score of 0 indicated normal patency; a score of 1, minimal-less than 50\%-stenosis; a score of 2 , one lesion with $50 \%$ or greater stenosis; a score of 3 , more than one lesion with $50 \%$ or greater stenosis; and a score of 4 , occlusion. In each patient, 31 anatomic segments of the lower extremity arteries were evaluated and scored (16).

Failure to visualize at nonenhanced MR angiography a segment that was depicted at contrast-enhanced MR angiography (the reference standard) was considered an incorrect assessment with the sequence. Segments that were not included in the imaging field of view at any sequence were excluded from analysis.

For all subjects, the diagnostic quality of images obtained at each of three imaging stations was graded by using a Likert scale (with grades of 0-4): Two radiologists (P.A.H., T.G.S.) independently assigned the nonenhanced and contrast-enhanced MR angiograms a score of 0 for nondiagnostic image; 1 for poor quality, with the observer not confident; 2 for fair quality, with the observer marginally confident; 3 for good quality, with the observer confident; or 4 for excellent quality, with the observer highly confident. An interventionalist (J.D.C.) who was blinded to the nonenhanced and contrast-enhanced MR angiogram findings and clinical histories read the MR angiograms first and then correlated these with the DSA images, when they existed. The limited number 
of patients who underwent DSA reflected the patients who underwent revascularization after contrast-enhanced MR angiography evaluation.

\section{Statistical Analyses}

The Wilcoxon signed rank test was used to assess the diagnostic performance of nonenhanced MR angiography, as compared with contrast-enhanced MR angiography, for the detection of significant $(\geq 50 \%)$ stenosis on a per-segment basis, per-region basis, and per-limb basis. For the per-region and per-limb analyses, the segment grades were averaged for each region (aorta, pelvis, thigh, and calf) and each limb. For the contrast-enhanced MR angiography protocol in the calves for which both boluschase and time-resolved MR angiography findings were available, the higher gradeindicating the more severe stenosiswas considered the diagnostic grade and was used in the analysis.

The sensitivity and specificity of QISS nonenhanced MR angiography, as compared with contrast-enhanced MR angiography (reference standard), for determination of nonsignificant $(<50 \%)$ versus significant $(50 \%-100 \%)$ stenosis by each reviewer were calculated per segment, per region, and per limb on the basis of simple proportions. For the per-region and per-limb calculations, the highest grade-indicating the most severe stenosis-was identified for the regions and limbs. The measures of sensitivity and specificity that we used reflected the performance of nonenhanced MR angiography versus contrastenhanced MR angiography separately, while the resulting mean summary measure, standard deviation, and confidence intervals (CIs) fully accounted for the correlations between adjacent stenoses per leg through generalized estimating equations (GEEs) with use of an unstructured binomial logit model (17). The sensitivity of nonenhanced or contrastenhanced MR angiography was defined as the probability of detecting a stenosis given that the patient has a stenosis at each arterial level, with the assumption that nonenhanced MR angiography and contrast-enhanced MR angiography theoretically will perform equally at each level. This did not preclude the fact that if a stenosis was found at a particular level, the likelihood of another stenosis would be higher with the GEE model. The GEE was used for the segment- and region-based analyses only; the limb-based analysis was considered to be independent. On the basis of the stenosis scores for one of the reviewers, QISS nonenhanced MR angiography and contrast-enhanced MR angiography were compared on a segment basis in those patients with GFRs of $60 \mathrm{~mL} /$ $\min / 1.73 \mathrm{~m}^{2}$ or lower $(n=17)$. In 15 patients, the same comparison was performed between conventional DSA, which served as the reference standard, and both nonenhanced and contrastenhanced MR angiography. Performance indexes of sensitivity and specificity were then compared between the patients with normal GFRs and those with low GFRs by using the $\chi^{2}$ test. Corresponding $95 \%$ CIs were provided for each estimate.

Likert scores of diagnostic image quality were compared by using the paired $t$ test and were reported as means \pm standard deviations. For assessment of interobserver variability, the agreement between the readers regarding the stenosis severity determined with each MR angiography technique was evaluated by using Cohen $\kappa$ values. A $\kappa$ value of 0.20 or lower implied poor agreement; values of $0.21-0.40$, fair agreement; values of $0.41-0.60$, moderate agreement; values of $0.61-0.80$, substantial agreement; and values of 0.81-1.00, almost perfect agreement (18). $P<.05$ was considered to indicate statistical significance. All statistical analyses were performed by using commercially available software (SPSS, version 18.0; SPSS, Chicago, III).

\section{Results}

Between March 2009 and March 2010, 53 patients (37 men, 16 women; mean age, 66.1 years; age range, 24-87 years) met the inclusion criteria for study participation, having undergone nonenhanced and contrast-enhanced MR angiography during the same examination. A total of 106 limbs, 348 regions, and 1696 seg- ments were evaluated for stenosis degree and diagnostic image quality in each segment. For reader 1, 480 segments had $50 \%$ or greater stenosis and 1084 segments had less than $50 \%$ stenosis at nonenhanced MR angiography, while 501 segments had $50 \%$ or greater stenosis and 1033 had less than 50\% stenosis at contrast-enhanced MR angiography (reference standard). One hundred thirty-two segments depicted at nonenhanced MR angiography and 162 segments depicted at contrast-enhanced MR angiography were diagnostically nonreadable for reader 1. For reader 2, $484 \mathrm{seg}$ ments had $50 \%$ or greater stenosis and 1073 had less than 50\% stenosis at nonenhanced MR angiography, while 502 segments had $50 \%$ or greater stenosis and 1030 segments had less than 50\% stenosis at contrast-enhanced MR angiography. One hundred thirty-nine segments depicted at nonenhanced MR angiography and 164 segments depicted at contrast-enhanced MR angiography segments were diagnostically nonreadable for reader 2 . The matched nonenhanced and contrast-enhanced MR angiography-depicted segments on nonreadable images were excluded owing to stent artifacts. Values of the diagnostic performance of nonenhanced MR angiography in the detection of lower extremity vascular stenosis are summarized in Table 2. Clinical examples are shown in Figures 2-6.

\section{Diagnostic Performance of Nonenhanced versus Contrast-enhanced MR Angiography}

Segment-based analysis. - In the pairwise comparison, nonenhanced and contrastenhanced MR angiography techniques yielded significantly similar stenosis grades in all but four of 16 segments (external iliac, distal superficial femoral, popliteal, and proximal peroneal arteries for reviewer 1; distal superficial femoral, proximal anterior tibial, proximal peroneal, and distal peroneal arteries for reader 2). Mean values of the diagnostic performance of nonenhanced MR angiography for the two reviewers without GEE modeling were $89.7 \%$ (436 of 486 segments) and $87.0 \%$ (423 of 486 segments) sensitivity and 
Table 2

\section{Diagnostic Performance of QISS Nonenhanced vs Contrast-enhanced MR Angiography}

\begin{tabular}{|c|c|c|c|c|c|c|}
\hline \multirow[b]{2}{*}{ Parameter } & \multicolumn{2}{|c|}{ Per Segment* } & \multicolumn{2}{|c|}{ Per Region* } & \multicolumn{2}{|c|}{ Per Limb ${ }^{\dagger}$} \\
\hline & Reviewer 1 & Reviewer 2 & Reviewer 1 & Reviewer 2 & Reviewer 1 & Reviewer 2 \\
\hline Sensitivity (\%) & $88.5(84.8,92.1)$ & $85.0(80.7,89.4)$ & $94.0(89.4,98.6)$ & $89.4(85.8,93.0)$ & $100(84 / 84)[95.7,100]$ & $100(84 / 84)[95.7,100]$ \\
\hline Specificity (\%) & $96.8(95.5,98.1)$ & $94.9(93.6,96.3)$ & $92.0(87.0,96.9)$ & $87.4(80.8,94.0)$ & $91(20 / 22)[70.8,98.8]$ & $91(20 / 22)[70.8,98.8]$ \\
\hline
\end{tabular}

Note.-Data are values of the diagnostic performance of nonenhanced MR angiography, as compared with contrast-enhanced MR angiography, in the detection of significant ( $\geq 50 \%$ ) stenosis.

* Per-segment and per-region analyses were performed with GEE modeling. Per-segment analysis was based on data from a total of 1516 segments. Per-region analysis was based on data from a total of 348 regions. Numbers in parentheses are $95 \%$ Cls.

† Per-limb analysis was based on data from a total of 106 limbs. Absolute numbers of limbs used to calculate the percentages are in parentheses, and $95 \%$ Cls are in brackets.

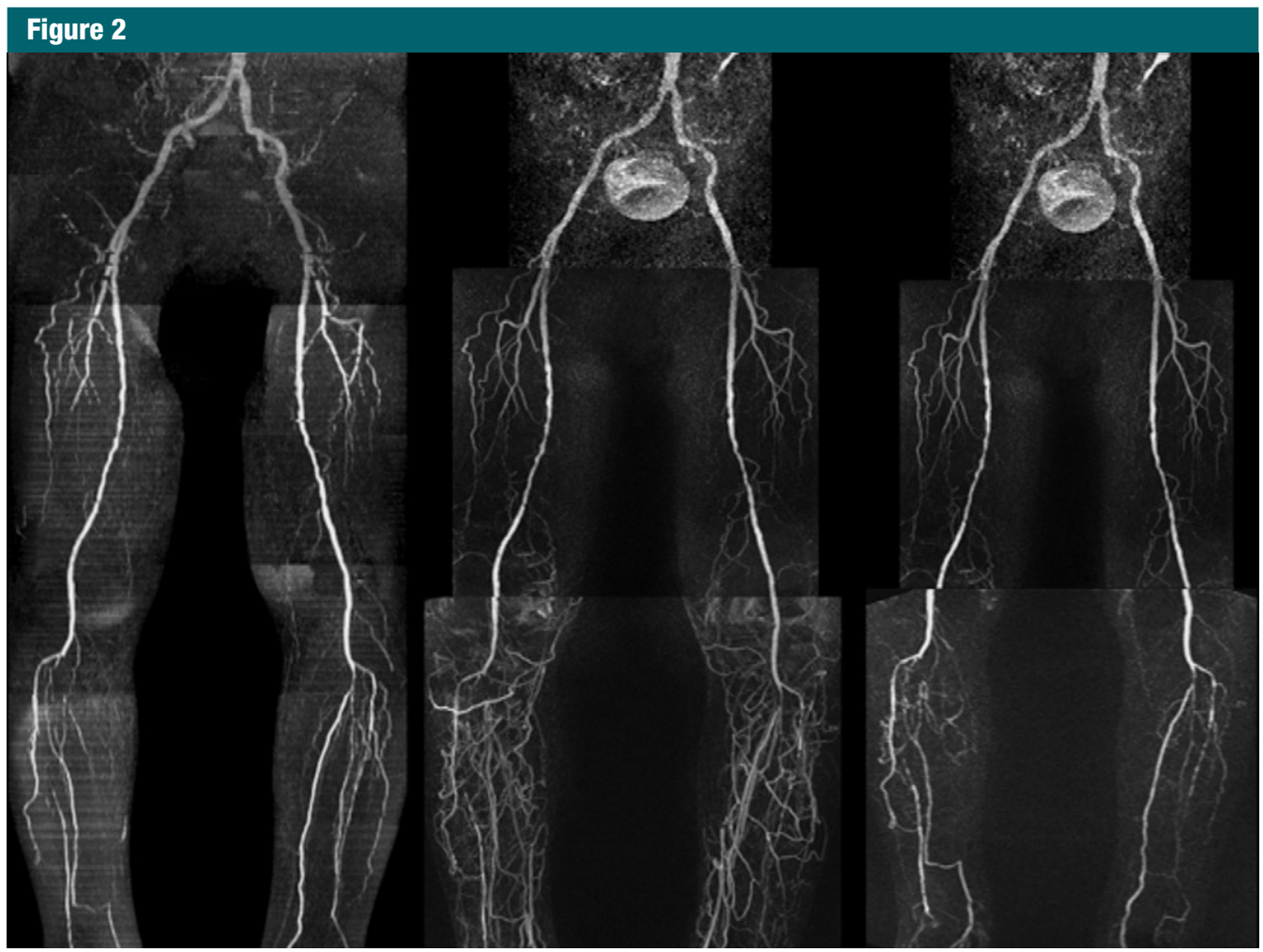

Figure 2: QISS nonenhanced (left), contrast-enhanced bolus-chase (middle), and time-resolved calf (right) MR angiograms show severe bilateral occlusive PAD below the knee. Although some horizontal artifacts are present on the QISS MR angiogram owing to atrial fibrillation and occasional electrocardiographic misgating, the image remains diagnostic.

$96.5 \%$ (994 of 1030 segments) and $94.6 \%$ (973 of 1028 segments) specificity. With GEE modeling, the patient-averaged population sensitivities of nonenhanced MR angiography for readers 1 and 2 were $88.5 \%$ (95\% CI: $84.8 \%, 92.1 \%)$ and $85.0 \%$ (95\% CI: $80.7 \%, 89.4 \%)$, respectively, and the patient-averaged population specificities were $96.8 \%(95 \%$ CI: $95.5 \%, 98.1 \%)$ and $94.9 \%$ (95\% CI: $93.6 \%, 96.3 \%)$, respectively.
Region-based analysis.-Nonenhanced MR angiography and contrastenhanced MR angiography yielded similar stenosis grades in all but one region (calf for reviewer 1, no region for reviewer 2). The sensitivities of nonenhanced MR angiography for the two readers with GEE modeling were 94.0\% (95\% CI: $89.4 \%, 98.6 \%)$ and $89.4 \%$ (95\% CI: $85.8 \%, 93.0 \%$ ), and the specificities were $92.0 \%$ (95\% CI:
$87.0 \%, 96.9 \%)$ and $87.4 \%(95 \% \mathrm{CI}$ : $80.8 \%, 94.0 \%)$.

Limb-based analysis.-No significant differences in diagnostic performance were detected between the two techniques at limb-based analysis. Mean values of the diagnostic performance of nonenhanced MR angiography were $100 \%$ (84 of 84 limbs) sensitivity and $91 \%$ (20 of 22 limbs) specificity. 


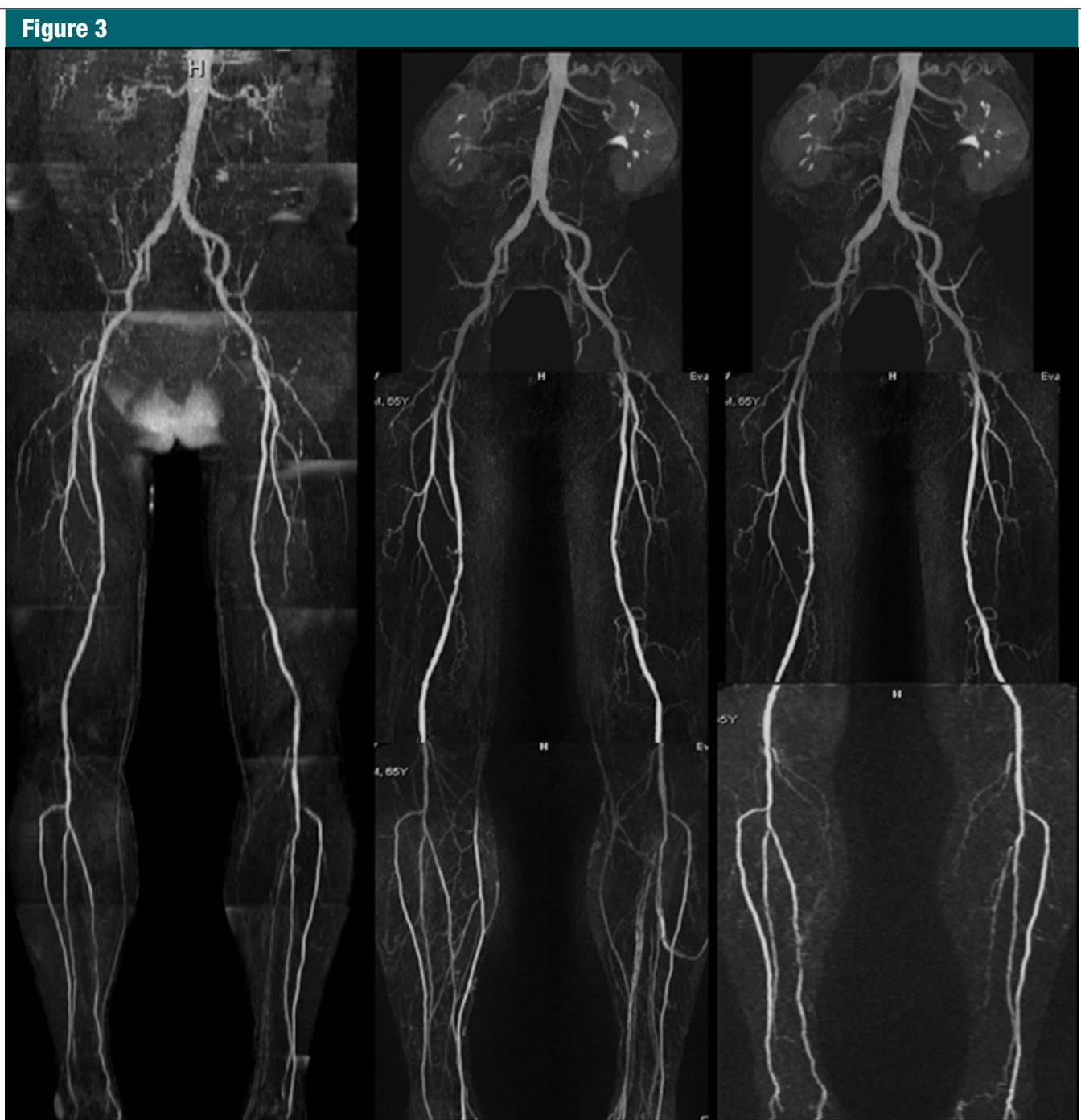

Figure 3: QISS nonenhanced (left), contrast-enhanced bolus-chase (middle), and time-resolved (right) MR angiograms show occlusion of left posterior tibial artery in patient with a nonhealing leg ulcer. Moderate- to high-grade multifocal stenosis of the middle portion of the right posterior tibial artery also is shown.

Diagnostic Performance of Nonenhanced MR Angiography in Patients with

\section{Compromised Renal Function}

On a per-segment basis, the values of the diagnostic performance of nonenhanced MR angiography in the 17 patients with GFRs of $60 \mathrm{~mL} / \mathrm{min} / 1.73 \mathrm{~m}^{2}$ or lower for the two readers were sensitivities of $83.5 \%$ and $86.0 \%$ and specificities of $92.3 \%$ and $95.0 \%$, which were comparable to the values in the patients with GFRs higher than $60 \mathrm{~mL} / \mathrm{min} / 1.73 \mathrm{~m}^{2}$ : sensitivities of $89.3 \%$ and $93.2 \%$ and specificities of $95.6 \%$ and $97.1 \%$ (Table 3). On a per-segment basis, a significant difference in sensitivity between the patients with normal renal function and those with compromised renal function (86.0\% vs $93.2 \%, P=.009$ ) was observed for reader 1; however, comparison of the specificities revealed no significant difference (95.0\% vs $97.1 \%, P=.086$ ). No significant differences in sensitivity or specificity were observed on a perregion or per-limb basis $(P>.05$ for all comparisons) (Table 3$)$.

\section{Comparison of Likert Scores for Diagnostic Image Quality}

The mean Likert score for contrastenhanced MR angiography was significantly higher in the pelvis $(3.58 \pm 0.69$ vs $3.19 \pm 0.84$ ) for reader 1 and in both the pelvis $(3.44 \pm 0.69$ vs $2.81 \pm 0.68)$ and the thigh $(3.49 \pm 0.72$ vs $3.27 \pm$ 0.69 ) for reader 2 . No significant differences were identified in other regions (Table 4).

\section{Nonenhanced MR Angiography versus Conventional DSA}

Selective catheter DSA was compared with QISS nonenhanced MR angiography in 15 patients (279 segments). Pairwise comparison of the stenosis grades revealed significant differences between the techniques in two of 16 segmentsin the profunda femoris and posterior 
tibial arteries. Mean values of the diagnostic performance of nonenhanced MR angiography were $91.0 \%$ (91 of 100 segments) sensitivity and 96.6\% (173 of 179 segments) specificity (Table 5).

DSA was compared with contrastenhanced MR angiography in the same 15 patients (262 segments). Significant differences were detected in three of 16 segments-in the common iliac, profunda femoris, and proximal posterior tibial arteries. Mean values of the diagnostic performance of contrast-enhanced MR angiography were $89 \%$ (86 of 97 segments) sensitivity and $95.8 \%$ (158 of 165 segments) specificity (Table 5 ).

\section{Interreader Agreement}

Almost perfect agreement between the two readers regarding stenosis scores was achieved, with Cohen $\kappa$ values of greater than 0.80 for all MR angiography techniques $(\kappa=0.92$ for contrastenhanced bolus-chase MR angiography, $\kappa=0.90$ for nonenhanced MR angiography, $\kappa=0.94$ for time-resolved angiography).

\section{Discussion}

Nonenhanced MR angiography performed with QISS is a rapid nonenhanced MR angiography examination, which involved a typical total imaging time of 6.4 minutes in our study. This imaging time compares favorably with that in older time-of-flight approaches, which could require 1 hour or longer. The short examination time helps to ensure patient compliance, especially since patients with PAD often have associated medical symptoms, such as back pain and leg discomfort, that make it difficult to remain immobile in a supine position for an extended period. Moreover, unlike with some other nonenhanced MR angiography techniques, with QISS, there is no need to calibrate the sequence parameters according to the flow pattern or severity of disease, making this technique easier to use.

In our study, nonenhanced MR angiography demonstrated excellent diagnostic performance in the detection of substantial PAD, with $85.0 \%-89.7 \%$ sensitivity and $94.6 \%-96.8 \%$ specific-

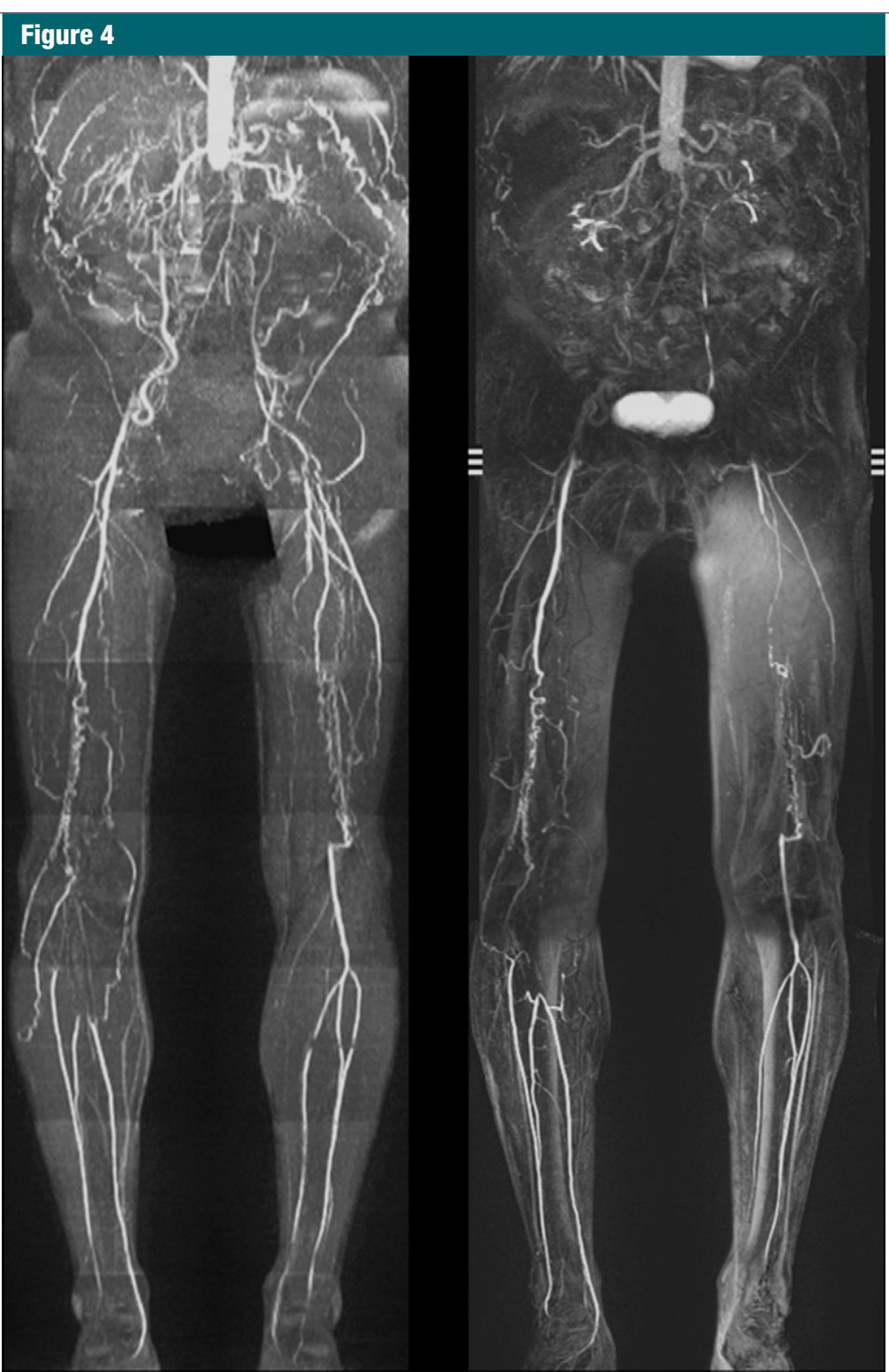

Figure 4: QISS nonenhanced (left) and contrast-enhanced bolus-chase (right) MR angiograms in patient who presented with thigh- and buttock-related claudication show bilateral aortoiliac occlusive disease. QISS nonenhanced and contrast-enhanced bolus-chase MR angiography findings show excellent correlation. Signal dropout extending horizontally across the proximal left trifurcation vessels may have been caused by electrocardiographic misgating.

ity compared with performance values for the combination of time-resolved and stepping-table bolus-chase contrastenhanced MR angiography. Nonenhanced MR angiography even had excellent diagnostic performance in a subgroup of patients when DSA was the reference standard, with $91.0 \%$ sensitivity and
96.6\% specificity. There was excellent interobserver agreement, with results that compared favorably with those of other nonenhanced MR angiography techniques (12).

The ankle brachial index usually is the test initially performed for hemodynamically significant PAD. However, 


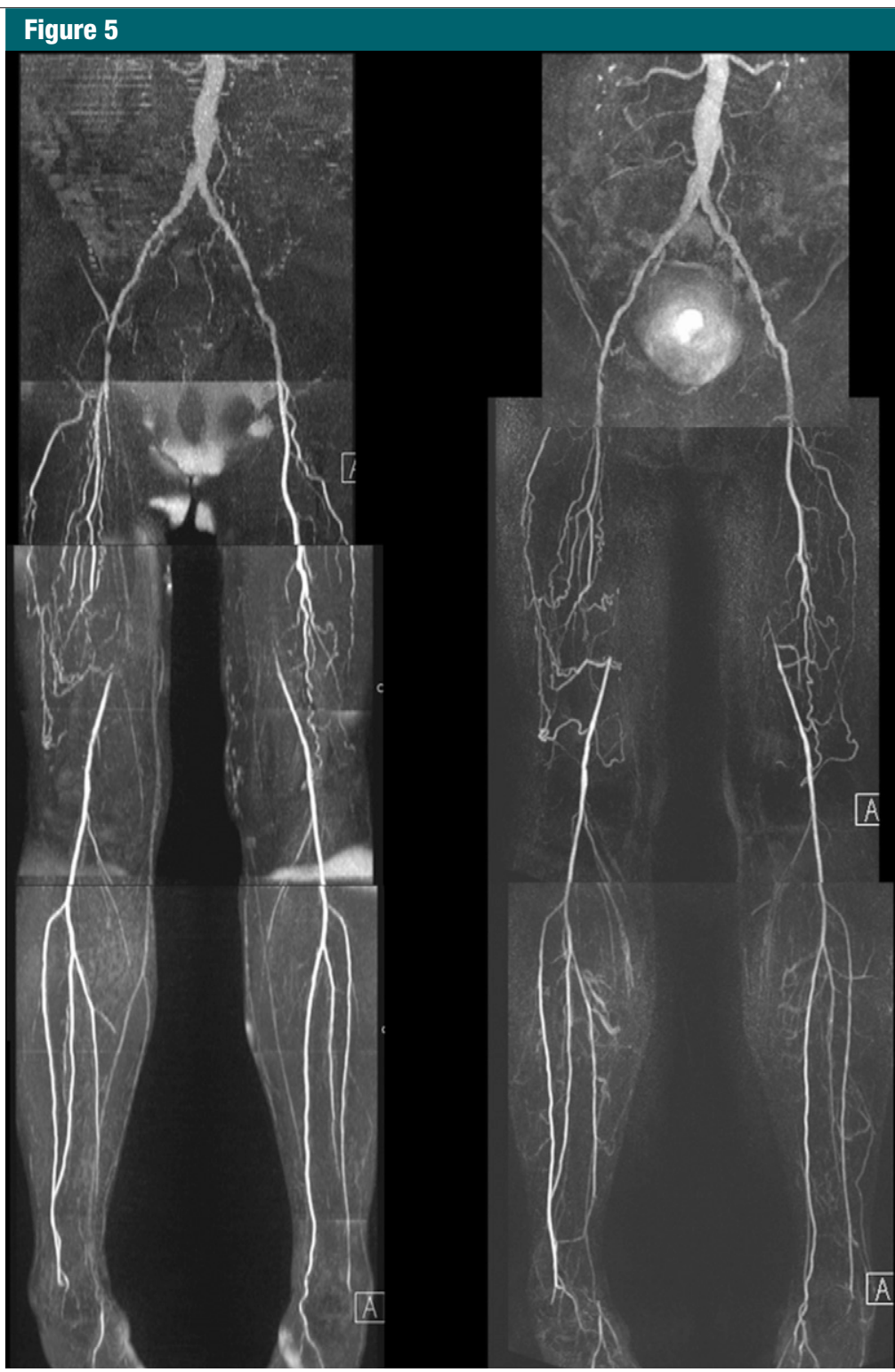

Figure 5: QISS nonenhanced (left) and contrast-enhanced bolus-chase (right) MR angiograms show occluded right and left superficial femoral arteries with collateral vessels.

because it does not enable accurate delineation of the location or severity of individual lesions, Doppler US may be used for further lesion characterization. Additional imaging typically is required before the revascularization. While DSA traditionally has been favored owing to high spatial resolution and reliable depiction of arterial disease, the rate of complications associated with DSA (4\%-9\%), including hematoma, anaphylaxis, arterial injury, and renal failure, as well as the associated costs, has resulted in a shift toward the use of contrastenhanced MR angiography and CT angiography. In the United States, DSA is largely reserved for use as an adjunctive diagnostic procedure performed immediately before or during revascularization (19).

Both CT angiography and contrastenhanced MR angiography enable accurate assessment of PAD in most patients and help guide treatment planning
$(20,21)$. Despite these advantages and the better spatial resolution afforded by using CT angiography, others have found the diagnostic confidence with CT angiography to be lower than that with DSA (22). The accuracy of DSA is diminished in diabetic patients because of the prevalence of calcified plaque, which is particularly troublesome in the evaluation of distal disease (23). Compared with CT angiography, contrast-enhanced MR angiography yields images with superior vessel contrast and no disturbing overlay from bone or calcified plaques. The rates of nondiagnostic and repeat CT angiography examinations increase with more advanced stages of disease (24). However, one does not have the opportunity to repeat either CT angiography or contrast-enhanced MR angiography when technical difficulties interfere with the diagnostic quality. To the contrary, nonenhanced MR angiography has the advantage that it can be repeated as needed. This was not done in the current study but would be expected to further improve the accuracy of the technique. Given that it only takes about 1 second to perform QISS MR angiography for any given section, repeating the nonenhanced MR angiography acquisition for sections with poor image quality would not be burdensome to the patient and would be expected to improve accuracy. If the patient's renal function permits, one can also supplement the nonenhanced MR angiography examination with low-dose contrastenhanced MR angiography should it prove necessary for better evaluation of a particular vessel segment.

Given the high prevalence of renal dysfunction and arterial calcification, QISS nonenhanced MR angiography might prove beneficial for diabetic patients with PAD (25). Poorly compressible calcified vessels may produce spurious results in diabetic and elderly patients, and investigators in ongoing studies are investigating the role of QISS nonenhanced MR angiography in diabetic patients with symptomatic PAD (26).

Our study had several limitations. It was not possible to completely blind the readers as to whether the MR angiography examination was contrast enhanced 
or nonenhanced because distinguishing features such as contrast agent in the bladder could not be hidden. The effect of the imaging test on the patient's clinical management was not assessed. The number of subjects was relatively small, and only two centers participated in the trial. In clinical practice, DSA has been supplanted by contrast-enhanced MR angiography and CT angiography for the preoperative evaluation of PAD. Consequently, in our study, DSA was only available as a reference standard in a subgroup of subjects, primarily those who were scheduled to undergo intervention. However, the combination of highspatial-resolution contrast-enhanced MR angiography and time-resolved imaging of the distal circulation, which was a reference standard in our study, has been shown to have accuracy comparable to that of DSA (27) and in some cases to enable more complete assessment. It is not known whether the severity of PAD in our study population was representative of the severity of disease in the general population of patients with PAD; the accuracy of the described nonenhanced MR angiography technique might vary depending on whether one is examining a population with milder disease or a population with more severe disease. Although direct head-to-head comparisons with other nonenhanced MR angiography techniques were outside the scope of our study design, it forms the basis for ongoing and prospective studies. The QISS sequence is not intrinsically directional. The directionality is derived from the application of the inferior tracking presaturation pulse. Thus, retrograde flow with retrograde filling of the proximal patent portion of an artery may not be routinely identified.

Future research directions include additional QISS MR angiography examinations with no tracking saturation pulse through the area of interest, which, in principle, may depict areas of retrograde flow. It should be noted that straightforward modifications of the QISS imaging protocol would be expected to improve the accuracy of the technique. For instance, since completing this study, we have altered the protocol to image the abdominal and upper

\begin{tabular}{|c|c|c|c|c|}
\hline \multicolumn{5}{|c|}{$\begin{array}{l}\text { Diagnostic Performance of Nonenhanced MR Angiography for Detection of Significant } \\
\text { Stenosis in Patients with Compromised vs Normal Renal Function }\end{array}$} \\
\hline \multirow[b]{2}{*}{ Parameter } & \multicolumn{2}{|c|}{$\mathrm{GFR} \leq 60 \mathrm{~mL} / \mathrm{min} / 1.73 \mathrm{~m}^{2}$} & \multicolumn{2}{|c|}{$\mathrm{GFR}>60 \mathrm{~mL} / \mathrm{min} / 1.73 \mathrm{~m}^{2}$} \\
\hline & Reviewer 1 & Reviewer 2 & Reviewer 1 & Reviewer 2 \\
\hline \multicolumn{5}{|l|}{ Per segment } \\
\hline Sensitivity & $\begin{array}{r}86.0(203 / 236) \\
{[80.9,90.1]}\end{array}$ & $\begin{array}{r}83.5(197 / 236) \\
{[78.1,87.9]}\end{array}$ & $\begin{array}{r}93.2(233 / 250) \\
{[89.3,95.9]}\end{array}$ & $\begin{array}{r}89.3(226 / 253) \\
{[84.8,92.8]}\end{array}$ \\
\hline Specificity & $\begin{array}{r}95.0(283 / 298) \\
{[91.8,97.1]}\end{array}$ & $\begin{array}{r}92.3(275 / 298) \\
{[88.6,95.0]}\end{array}$ & $\begin{array}{c}97.1(711 / 732) \\
{[95.6,98.2]}\end{array}$ & $\begin{array}{r}95.6(698 / 730) \\
{[93.8,96.9]}\end{array}$ \\
\hline \multicolumn{5}{|l|}{ Per region } \\
\hline Sensitivity & $\begin{array}{l}94(68 / 72) \\
\quad[86.3,98.4]\end{array}$ & $\begin{array}{l}89(65 / 73) \\
\quad[79.5,95.1]\end{array}$ & $\begin{array}{c}97.2(103 / 106) \\
{[91.9,99.4]}\end{array}$ & $\begin{array}{r}92.4(97 / 105) \\
{[85.5,96.6]}\end{array}$ \\
\hline Specificity & $\begin{array}{l}96(45 / 47) \\
\quad[85.4,99.4]\end{array}$ & $\begin{array}{l}91(42 / 46) \\
\quad[79.2,97.5]\end{array}$ & $\begin{array}{r}93.6(117 / 125) \\
{[87.7,97.2]}\end{array}$ & $\begin{array}{r}89.7(113 / 126) \\
{[83.0,94.3]}\end{array}$ \\
\hline \multicolumn{5}{|l|}{ Per limb } \\
\hline Sensitivity & $\begin{array}{c}100(32 / 32) \\
{[91.0,100]}\end{array}$ & $\begin{array}{c}100(32 / 32) \\
{[91.0,100]}\end{array}$ & $\begin{array}{c}100(52 / 52) \\
{[94.4,100]}\end{array}$ & $\begin{array}{c}100(52 / 52) \\
{[94.4,100]}\end{array}$ \\
\hline Specificity & $\begin{array}{l}100(2 / 2) \\
{[22.3,100]}\end{array}$ & $\begin{array}{l}100(2 / 2) \\
{[22.3,100]}\end{array}$ & $\begin{array}{l}90.0(18 / 20) \\
{[68.3,98.7]}\end{array}$ & $\begin{array}{l}90.0(18 / 20) \\
{[68.3,98.7]}\end{array}$ \\
\hline
\end{tabular}

Note.-All data are percentages, with numbers used to calculate the percentages in parentheses and $95 \%$ Cls in brackets Values were derived from a comparison of the diagnostic performance of nonenhanced MR angiography in the detection of significant ( $\geq 50 \%$ ) stenosis between patients with compromised renal function (GFR $\leq 60 \mathrm{~mL} / \mathrm{min} / 1.73 \mathrm{~m}^{2}$ ) and patients with normal renal function (GFR $>60 \mathrm{~mL} / \mathrm{min} / 1.73 \mathrm{~m}^{2}$ ).

\section{Table 4}

Comparison of Likert Scores for Diagnostic Image Quality

\begin{tabular}{|c|c|c|c|c|c|c|}
\hline \multirow{2}{*}{$\begin{array}{l}\text { MR Angiography } \\
\text { Sequence }\end{array}$} & \multicolumn{2}{|c|}{ Pelvis } & \multicolumn{2}{|c|}{ Thigh } & \multicolumn{2}{|c|}{ Calf } \\
\hline & Reviewer 1 & Reviewer 2 & Reviewer 1 & Reviewer 2 & Reviewer 1 & Reviewer 2 \\
\hline Nonenhanced & $3.19 \pm 0.84$ & $2.81 \pm 0.68$ & $3.46 \pm 0.69$ & $3.27 \pm 0.69$ & $3.33 \pm 0.70$ & $3.20 \pm 0.70$ \\
\hline Contrast enhanced & $3.58 \pm 0.69$ & $3.44 \pm 0.69$ & $3.47 \pm 0.72$ & $3.49 \pm 0.72$ & $3.34 \pm 1.04$ & $3.33 \pm 0.76$ \\
\hline$P$ value & .005 & $<.001$ & .99 & .029 & .566 & .087 \\
\hline
\end{tabular}

Note.-Data are mean Likert scores \pm standard deviations, compared between contrast-enhanced MR angiography and nonenhanced MR angiography in the pelvis, thigh, and calf regions. $P$ values were derived by using the paired $t$ test.

\section{Table 5}

\section{Comparison of DSA and MR Angiography Techniques}

\begin{tabular}{llc} 
Parameter & DSA vs QISS MR Angiography & DSA vs Contrast-enhanced MR Angiography \\
\hline Sensitivity & $91.0(91 / 100)[83.6,95.8]$ & $89(86 / 97)[80.6,94.2]$ \\
Specificity & $96.6(173 / 179)[92.8,98.7]$ & $95.8(158 / 165)[91.4,98.2]$
\end{tabular}

Note.-All data are percentages, with numbers used to calculate the percentages in parentheses and $95 \%$ Cls in brackets. Values were derived from comparisons of the diagnostic performance of DSA and various MR angiography techniques for the detection of significant $(\geq 50 \%)$ stenosis.

pelvic stations with a reduced number of sections per station. This allows the routine use of breath holding to eliminate respiratory motion artifacts. In addition, the section thickness has been decreased from 3.0 to $1.2 \mathrm{~mm}$ for the calf region, substantially improving the delineation of the trifurcation origins 


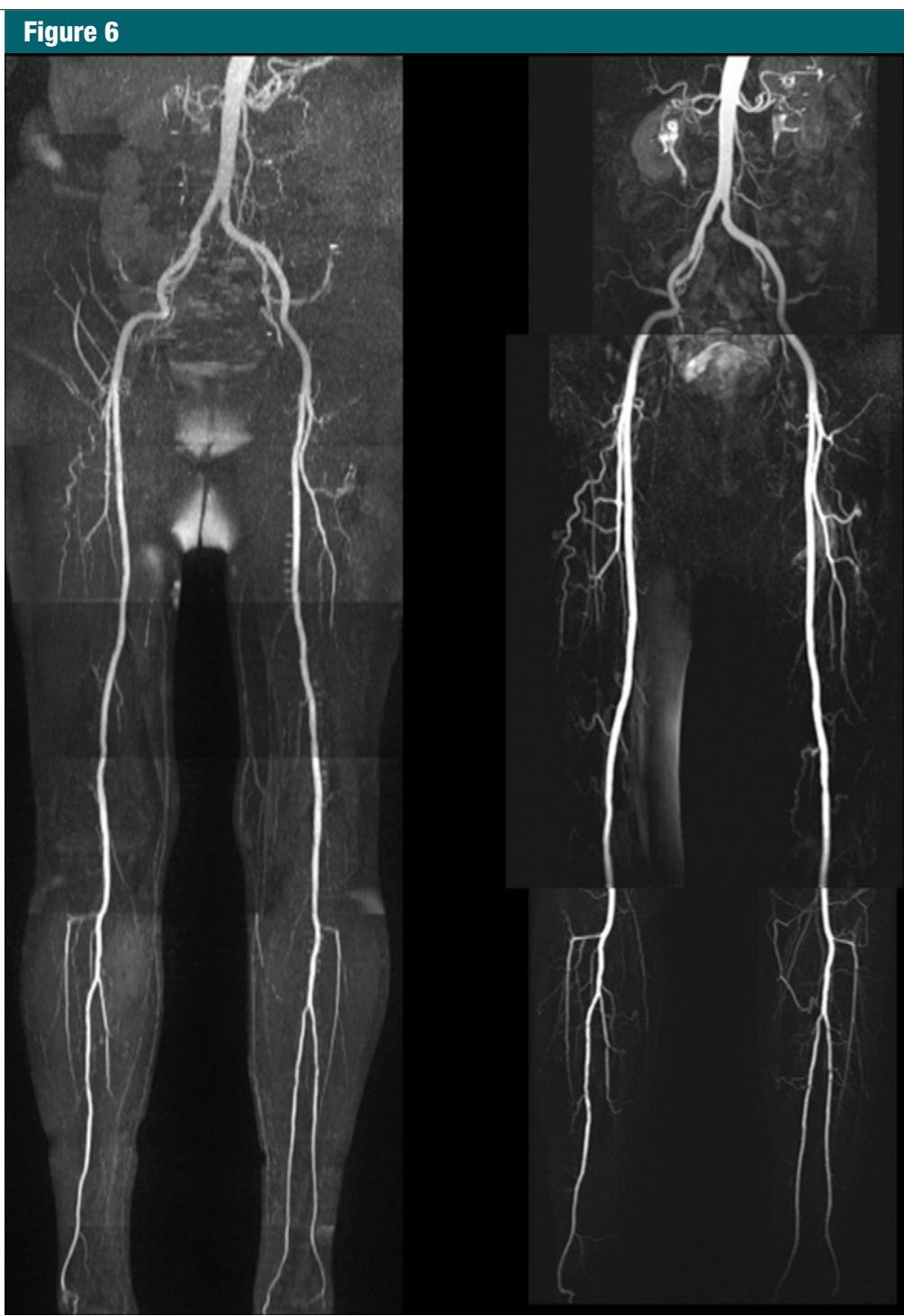

Figure 6: QISS nonenhanced (left) and contrast-enhanced bolus-chase (right) MR angiograms in patient who presented with left calf pain and had abnormal ankle brachial index show moderate multifocal stenoses of the left peroneal artery, with excellent correlation between QISS nonenhanced and contrast-enhanced bolus-chase MR angiography findings. Occluded right posterior tibial artery is also demonstrated.

and branch vessels. In conclusion, QISS nonenhanced MR angiography offers an alternative to currently used imaging tests for symptomatic chronic lower limb ischemia, in which the administration of iodinated or gadolinium-based contrast agents is contraindicated.

Acknowledgment: We are grateful to Asad Usman, MD, for assistance with the statistical analyses.

Disclosures of Potential Conflicts of Interest: P.A.H. No potential conflicts of interest to dis- close. I.K. No potential conflicts of interest to disclose. A.H.D. No potential conflicts of interest to disclose. T.G.S. No potential conflicts of interest to disclose. J.D.C. No potential conflicts of interest to disclose. J.J.S. No potential conflicts of interest to disclose. E.E.D. No potential conflicts of interest to disclose. N.G. No potential conflicts of interest to disclose. J.C.C. Financial activities related to the present article: none to disclose. Financial activities not related to the present article: received a research grant from Siemens Healthcare. Other relationships: none to disclose. R.R.E. Financial activities related to the present article: none to disclose. Financial activities not related to the present article: pat- ent application submitted for QISS technique; received royalties from Siemens Healthcare. Other relationships: none to disclose.

\section{References}

1. Leng GC, Lee AJ, Fowkes FG, et al. Incidence, natural history and cardiovascular events in symptomatic and asymptomatic peripheral arterial disease in the general population. Int J Epidemiol 1996;25(6):1172-1181.

2. Selvin E, Erlinger TP. Prevalence of and risk factors for peripheral arterial disease in the United States: results from the National Health and Nutrition Examination Survey, 1999-2000. Circulation 2004;110(6):738-743.

3. Hirsch AT, Haskal ZJ, Hertzer NR, et al. ACC/AHA 2005 practice guidelines for the management of patients with peripheral arterial disease (lower extremity, renal, mes enteric, and abdominal aortic): a collabora tive report from the American Association for Vascular Surgery/Society for Vascular Surgery, Society for Cardiovascular Angiography and Interventions, Society for Vascular Medicine and Biology, Society of Interventional Radiology, and the ACC/AHA Task Force on Practice Guidelines (Writing Committee to Develop Guidelines for the Management of Patients With Peripheral Arterial Disease)-endorsed by the American Association of Cardiovascular and Pulmonary Rehabilitation; National Heart, Lung, and Blood Institute; Society for Vascular Nursing; TransAtlantic Inter-Society Consensus; and Vascular Disease Foundation. Circulation 2006;113(11):e463-e654.

4. Lin PH, Bechara C, Kougias P, Huynh TT, LeMaire SA, Coselli JS. Assessment of aortic pathology and peripheral arterial disease using multidetector computed tomographic angiography. Vasc Endovascular Surg 2008 Dec-2009;42(6):583-598.

5. O'Hare AM. Management of peripheral arterial disease in chronic kidney disease. Cardiol Clin 2005;23(3):225-236.

6. Koelemay MJ, Lijmer JG, Stoker J, Legemate DA, Bossuyt PM. Magnetic resonance angiography for the evaluation of lower extremity arterial disease: a meta-analysis. JAMA 2001;285(10):1338-1345.

7. Baum RA, Rutter CM, Sunshine JH, et al Multicenter trial to evaluate vascular magnetic resonance angiography of the lower extremity: American College of Radiology Rapid Technology Assessment Group. JAMA 1995;274(11):875-880.

8. Kuo PH, Kanal E, Abu-Alfa AK, Cowper SE. Gadolinium-based MR contrast agents and nephrogenic systemic fibrosis. Radiology 2007;242(3):647-649. 
9. Owen RS, Carpenter JP, Baum RA, Perloff LJ, Cope C. Magnetic resonance imaging of angiographically occult runoff vessels in peripheral arterial occlusive disease. $\mathrm{N}$ Engl J Med 1992;326(24):1577-1581.

10. Weadock WJ, Chenevert TL. Emerging concepts in MR angiography: preface. Magn Reson Imaging Clin N Am 2009;17(1):xi.

11. Miyazaki M, Sugiura S, Tateishi F, Wada H, Kassai Y, Abe H. Non-contrast-enhanced MR angiography using 3D ECG-synchronized halfFourier fast spin echo. J Magn Reson Imaging 2000;12(5):776-783

12. Miyazaki M, Lee VS. Nonenhanced MR angiography. Radiology 2008;248(1):20-43.

13. Edelman RR, Sheehan JJ, Dunkle E, Schindler N, Carr J, Koktzoglou I. Quiescentinterval single-shot unenhanced magnetic resonance angiography of peripheral vascular disease: technical considerations and clinical feasibility. Magn Reson Med 2010;63(4): 951-958.

14. Pereles FS, Collins JD, Carr JC, et al. Accuracy of stepping-table lower extremity MR angiography with dual-level bolus timing and separate calf acquisition: hybrid peripheral MR angiography. Radiology 2006;240(1): 283-290.

15. Korosec FR, Frayne R, Grist TM, Mistretta CA. Time-resolved contrast-enhanced 3D MR angiography. Magn Reson Med 1996;36(3): 345-351.

16. Meaney JF, Ridgway JP, Chakraverty S, et al. Stepping-table gadolinium-enhanced digital subtraction MR angiography of the aorta and lower extremity arteries: preliminary experience. Radiology 1999;211(1):59-67.

17. Drake C, Levine RA. Sensitivity, specificity and other diagnostic measures with multiple sites per unit. Contemp Clin Trials 2005; 26(2):252-259.

18. Landis JR, Koch GG. The measurement of observer agreement for categorical data. Biometrics 1977;33(1):159-174.

19. Waugh JR, Sacharias N. Arteriographic complications in the DSA era. Radiology 1992; 182(1):243-246.

20. Khilnani NM, Winchester PA, Prince MR, et al. Peripheral vascular disease: combined 3D bolus chase and dynamic 2D MR angiography compared with x-ray angiography for treatment planning. Radiology 2002;224(1): 63-74.

21. Ouwendijk R, Kock MC, Visser K, Pattynama PM, de Haan MW, Hunink MG. Interobserver agreement for the interpretation of contrast-enhanced 3D MR angiography and MDCT angiography in peripheral arterial disease. AJR Am J Roentgenol 2005;185(5): 1261-1267.
22. Adriaensen ME, Kock MC, Stijnen T, et al Peripheral arterial disease: therapeutic confidence of CT versus digital subtraction angiography and effects on additional imaging recommendations. Radiology 2004;233(2): 385-391.

23. Ouwendijk R, Kock MC, van Dijk LC, van Sambeek MR, Stijnen T, Hunink MG. Vessel wall calcifications at multi-detector row CT angiography in patients with peripheral arterial disease: effect on clinical utility and clinical predictors. Radiology 2006;241(2): 603-608.

24. Dinter DJ, Neff KW, Visciani G, et al. Peripheral bolus-chase MR angiography: analysis of risk factors for nondiagnostic image quality of the calf vessels-a combined retrospective and prospective study. AJR Am J Roentgenol 2009;193(1):234-240.

25. Murabito JM, D'Agostino RB, Silbershatz H, Wilson WF. Intermittent claudication: a risk profile from the Framingham Heart Study. Circulation 1997;96(1):44-49.

26. American Diabetes Association. Peripheral arterial disease in people with diabetes. Diabetes Care 2003;26(12):3333-3341.

27. Meissner OA, Rieger J, Weber C, et al. Critical limb ischemia: hybrid MR angiography compared with DSA. Radiology 2005;235(1): 308-318. 